\title{
Histological disorders of neurons of phylogenetically different parts of the cerebral cortex in partial, subtotal, stepwise subtotal, and total cerebral ischemia
}

\author{
Lizaveta I. Bon \\ Grodno State Medical University, Grodno, Belarus \\ (iD) https://orcid.org/0000-0001-7189-0838 \\ Corresponding author: asphodela@list.ru
}

\section{Nataliya Y. Maksimovich}

Department of Pathological Physiology named after D.A. Maslakov, Grodno State Medical University, Grodno, Belarus (DD) https://orcid.org/0000-0003-3181-9513

DOI: https://doi.org/10.20883/medical.e493

\author{
Published: 2021-03-24
}

How to Cite: Bon LI, Maksimovich NY. Histological disorders of neurons of phylogenetically different parts of the cerebral cortex in partial, subtotal, stepwise subtotal, and total cerebral ischemia. JMS [Internet]. 2021 Mar 24;90(1):e493. doi:10.20883/medical.e493

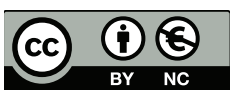

(c) 2021 by the author(s). This is an open access article distributed under the terms and conditions of the Creative Commons Attribution (CC BY-NC) licencse. Published by Poznan University of Medical Sciences

Keywords: cellular immunity, cytokines, leishmaniasis, pro-inflammation, HIV co-infection

\section{ABSTRACT}

Aim. Measure of the histological changes in neurons in the parietal cortex and hippocampus of rats with partial, subtotal, stepwise subtotal, and total cerebral ischemia.

Material and Methods. Studies were performed on 84 rats. Partial cerebral ischemia was modelled by ligation of one common carotid artery. Subtotal cerebral ischemia was modelled by ligation of both common carotid arteries. Stepwise subtotal cerebral ischemia was performed by sequential ligation of both common carotid artery with 7-day, 3-day or 1-day intervals. Total cerebral ischemia (CI) was modelled by decapitation.

Results. When comparing the morphological changes of neurons in the parietal cortex and hippocampus, we observed that, with the aggravation of the severity of cerebral ischemia, there was a progressive increase in the number of hyperchromic shrivelled neurons and neurons with pericellular oedema. Modelling of more severe types of ischemic damage lead to pronounced morphological changes in neurons - a decrease in size, deformation of the perikaryon, and increase in the degree of neuronal chromatophilia with their wrinkling. Conclusions. The smallest morphological changes in neurons were noted in the partial cerebral ischemia groups and subgroup 1 of stepwise subtotal cerebral ischemia, with an interval between common carotid artery dressings of 7 days. The most obvious morphological changes were observed in the conditions of total cerebral ischemia after 1 day. Changes in the parietal cortex and hippocampus were unidirectional, but in the parietal cortex, which is most sensitive to oxygen deficiency, they were more pronounced.

\section{Introduction}

The study of the brain in health and disease is an urgent and promising area of modern science and, in this regard, a frequent topic of dissertation research. Cerebrovascular and cardiovascular diseases are the most pressing medical and social problems in the world. Each year 450,000 
people suffer a stroke, of which $75-80 \%$ are ischemic strokes. Cerebral ischemia leads to a number of general and local metabolic and functional disorders, the pathogenesis of which is complex, multifaceted, and largely unclear [1-5]. Adequate models of cerebral pathology may contribute to the detailed understanding of the pathogenesis of these disorders and, in addition, allow assessing the development of damage and adaptive mechanisms of the brain, which serves as a fundamental basis for improving the diagnosis, treatment, and prevention. In previous studies, histological disorders of neurons of phylogenetically different parts of the cerebral cortex were studied in partial, subtotal, total, and subtotal stepwise cerebral ischemia. However, it is important to analyse these disorders in a comparative aspect.

\section{Aim}

The aim of the study was to compare histological changes in neurons in the parietal cortex and hippocampus of rats with partial, subtotal, subtotal stepwise, and total cerebral ischemia.

\section{Material and Methods}

The experiments were performed on 84 male outbred white rats weighing $240 \pm 20 \mathrm{~g}$ in compliance with the Directive of the European Parliament and Council No. 2010/63 / EU of 22.09.2010 on the protection of animals used for scientific purposes. The animals were kept in an air-conditioned room $\left(22{ }^{\circ} \mathrm{C}\right)$ under mixed illumination on a standard vivarium ration with free access to food and water, and no more than five animals per cage. Protocols were reviewed and approved by the Ethical Committee of the Grodno State Medical University (protocol No 1, 14.04.2013). Studies were performed on animals represented by five groups of 6 rats each. Partial cerebral ischemia (PCl) was modelled by ligation of one common carotid artery (CCA). Subtotal cerebral ischemia (SCI) was modelled by ligation of both CCA. Graded subtotal cerebral ischemia (SSCI) was performed by sequential ligation of both CCA with 7-day (subgroup 1), 3-day (subgroup 2) or 1-day (subgroup 3) intervals. Lastly, total cerebral ischemia (TCl) was modelled by decapitation. Spec- imens were divided into two groups and kept at $+16^{\circ} \mathrm{C}$. The brain was harvested 1 hour and 1 day after ligation of the CCA in case of $\mathrm{PCl}$ or the second CCA in case of $\mathrm{SCl}$ and $\mathrm{SSCl}$, or decapitation in case of $\mathrm{TCl}$ in each of the groups.

The control group consisted of sham-operated rats. The sampling of the material was carried out at a similar time after the sham operation. Changes in the size (area) and shape (form factor, elongation factor) of the neurons, as well as the degree of chromatophilia of their cytoplasm were compared with $\mathrm{Cl}$ for 1 hour and 1 day. After decapitation, the brain was quickly removed and pieces of the cerebral cortex were fixed in Carnoy's fluid. Paraffin sections ( $5 \mu \mathrm{m}$ thick) were prepared using a microtome (Leica RM 2125 RTS, Germany) and mounted on glass slides. The localisation of the parietal cortex and hippocampus of the cortex in the histological preparations of the brain was determined using a stereotaxic atlas [6]. Serial paraffin sections were stained with $0.1 \%$ toluidine blue according to the Nissl method.

Study of histological preparations, their microphotography, morphometry and densitometry of the chromogen sediment were conducted using an Axioscop 2 plus microscope (Zeiss, Germany), a digital video camera (LeicaDFC 320, Germany) and ImageWarp image analysis software (Bitflow, USA). In each animal, at least 30 neurons of the fifth layer of the parietal cortex and the pyramidal layer of the $\mathrm{CA}_{1}$ field of the hippocampus were assessed, which provided a sufficient sample size for subsequent analysis. To assess the severity of ischemic damage to the cerebral cortex under $\mathrm{Cl}$ conditions, changes in the size and shape of neuronal perikaryons, as well as the degree of colour of their cytoplasm (chromatophilia), were studied. The change in the size of neurons was assessed on the basis of their area, the shape of the perikaryons of neurons was assessed using the form factor $\left(4 \pi S / P^{2}-\right.$ the index of sphericity and folding), and the elongation factor - the index of sphericity (Dmax / Dmin) using the ImageWarp image analysis program (Bitflow, USA).

The area of neurons (S) and the elongation of their bodies indicated the state of the cytoskeleton and the water-electrolyte balance, which can be disturbed during cerebral ischemia. In the histological preparations, the number of pyramidal neurons per unit area of the cerebral cortex sections was determined. 
Among the total number of neurons, cells were distinguished according to the intensity of the colour of the cytoplasm (chromatophilia): normochromic - moderately coloured; hyperchromic - dark; hyperchromic wrinkled - very dark, with deformed perikaryon; hypochromic pale-coloured; shadow cells - unstained, with vacuolated nuclei; cells with pericellular oedema are shrunken, with enlightenment around the perikaryon. The number of each type of cells was counted.

A Kruskal-Wallis test with Bonferroni correction was applied to analyse the data (Statistica 10.0 software for Windows, StatSoft, Inc., USA). The results are presented in the form Me (LQ; $\mathrm{UQ}$ ), where $\mathrm{Me}$ is the median, $\mathrm{LQ}$ is the boundary of the lower quartile; $U Q$ is the boundary of the upper quartile. The differences between groups were considered significant at $p<0.05$.

\section{Results}

Compared with the control, there were no changes in the size and shape of the neurons perikaryon after 1 hour with $\mathrm{PCl}$ (Figure 1). However, in this group, both in the parietal cortex and hippocampus, there was a decrease of $18 \%(p<0.05)$ and $16 \%(p<0.05)$ in the number of normochromic neurons, as well as an increase of $65 \%(p<0.05)$ and $69 \%(p<0.05)$ in the number of hyperchromic neurons, respectively (Figures 2-4).

The absence of pronounced morphological changes in the simulation of $\mathrm{PCl}$ in rats was explained by the compensation of blood circulation along the circle of Willis. There were no differences in the severity of the changes noted in the parietal cortex and hippocampus. In animals with 1-hour SCl, the size of the neurons of the parietal cortex and hippocampus were $52 \%$

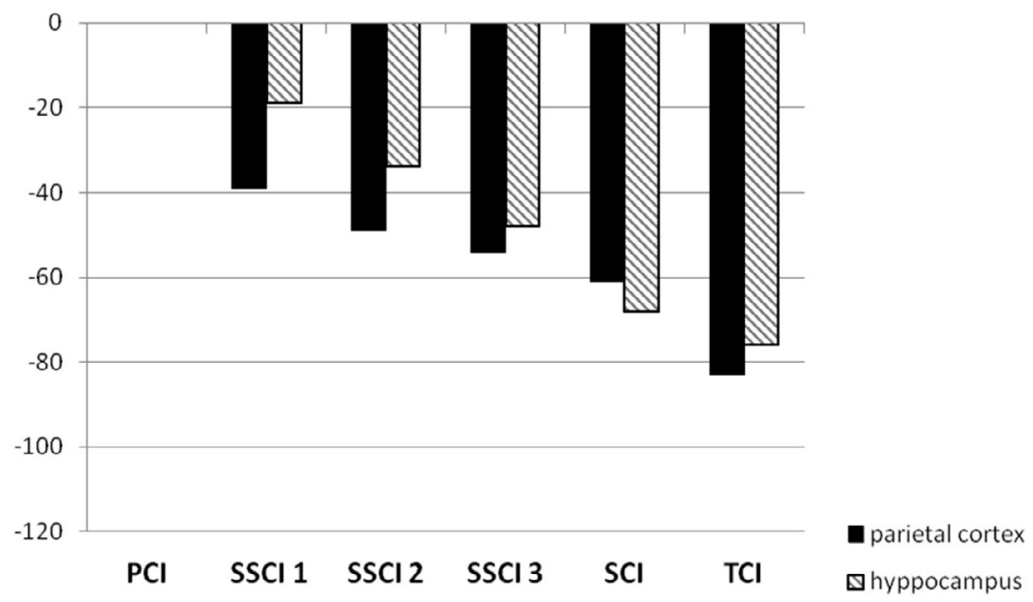

Figure 1. Changes in the area of perikaryon neurons in rats with cerebral ischemia $(\mathrm{Cl})$

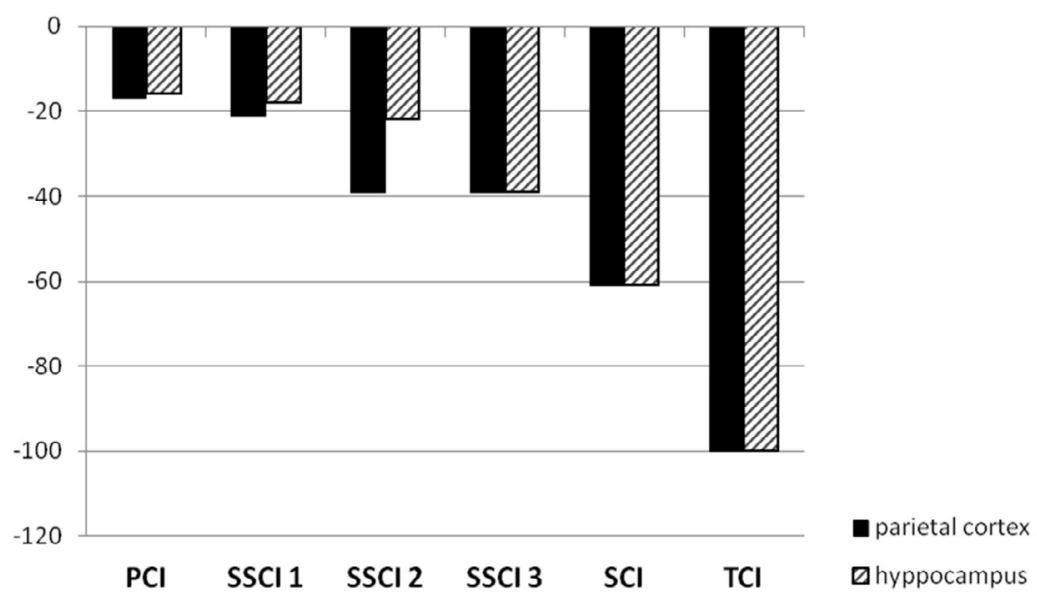

Figure 2. Changes in the number of normochromic neurons in rats with cerebral ischemia 
$(p<0.05)$ and $48 \%(p<0.05)$ smaller than in the control group, respectively. The elongation factor was $20 \%$ higher $(p<0.05)$ in both studied regions, and the form factor decreased by $11 \%(p<0.05)$ and $22 \%(p<0.05)$ in the parietal cortex and hippocampus, respectively. Compared with the $\mathrm{PCl}$ group, the area of the neurons in rats with $\mathrm{SCl}$ decreased by $57 \%$ in the parietal cortex $(\mathrm{p}<0.05)$ and $42 \%$ in the hippocampus $(p<0.05)$. At the same time, the elongation of neuronal perikaryons (elongation factor) increased by $20 \%(p<0.05)$ in each of the cortex sections, and their roundness (form factor) decreased by $11 \%$ in the parietal cortex $(p<0.05)$ and by $22 \%$ in the hippocam-
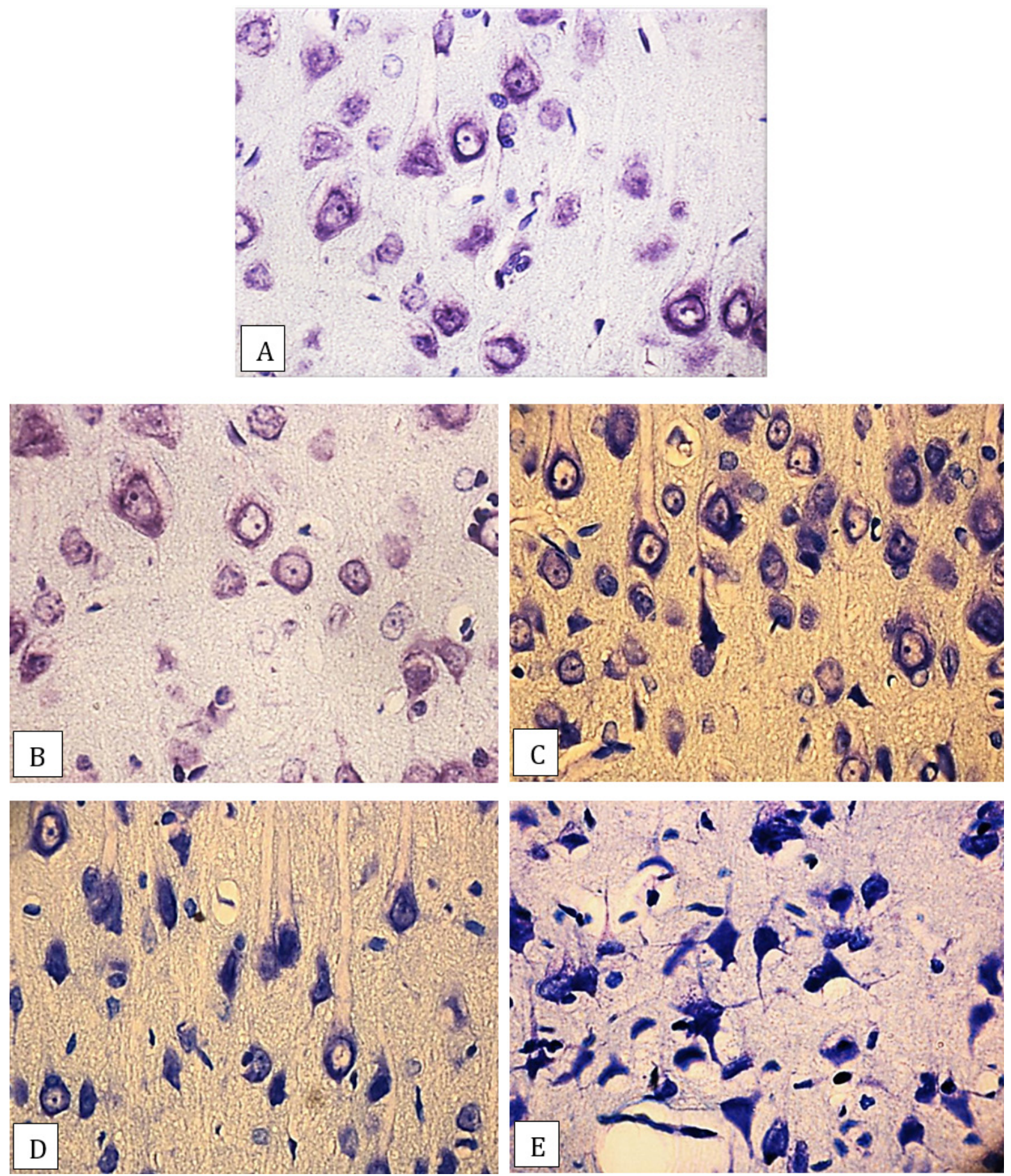

Figure 3. Neurons of the fifth layer of the parietal cortex in rats with a duration of the ischemic period of 1 hour. A - control, B - PCl (predominance of normochromic neurons), $\mathrm{C}-1^{\text {st }}$ subgroup of SSCI (predominance of hyperchromic neurons), D - SCI (predominance of hyperchromic and hyperchromic wrinkled neurons), E - TCI (absence of normochromic neurons, predominance of hyperchromic wrinkled neurons). Stained by Nissl. Digital microphotography. Scale bars and magnifications: A - $20 \mu \mathrm{m}$ and x 400 


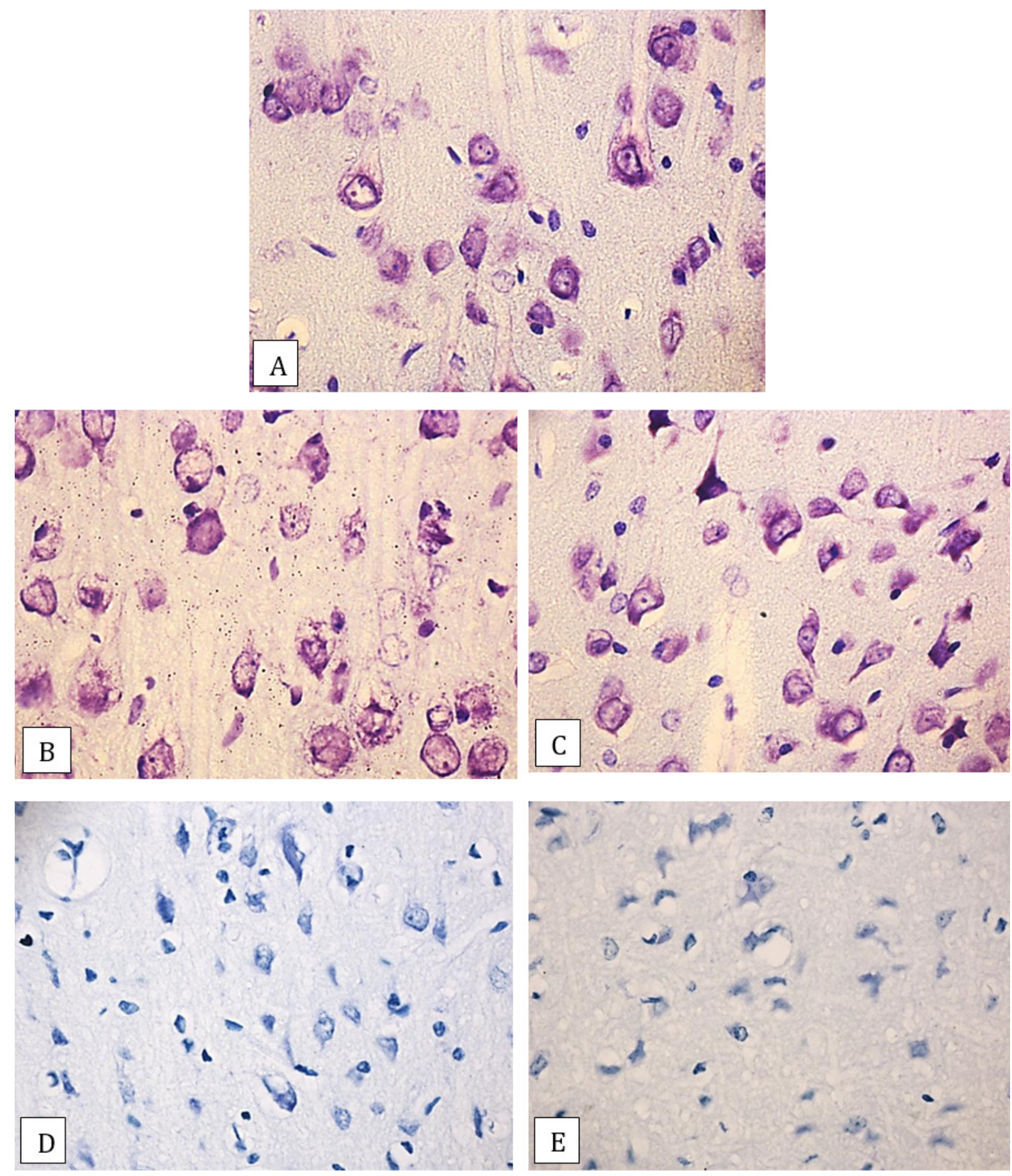

Figure 4. Neurons of the fifth layer of the parietal cortex in rats with a duration of the ischemic period of 1 day. A - control, B - PCI (predominance of normochromic neurons), $\mathrm{C}-1^{\text {st }}$ subgroup of $\mathrm{SSCl}$ (predominance of hyperchromic neurons), D - SCl (predominance of hyperchromic and hyperchromic wrinkled neurons), E - TCl (absence of normochromic neurons, predominance of hyperchromic wrinkled neurons). Stained by Nissl. Digital microphotography. Scale bars and magnifications: A - $20 \mu \mathrm{m}$. x 400

pus $(p<0.05)$. When the ischemic period lasted 1 day, the disturbances were aggravated; compared with the control group, the area of the neurons decreased by $61 \%(p<0.05)$ in the parietal cortex and $68 \%(p<0.05)$ in the hippocampus. These changes were much more pronounced than in the group "PCl" lasting 1 day, where the area of neurons in the group "SCl" decreased by $53 \%$ in the parietal cortex $(p<0.05)$ and $61 \%$ in the hippocampus $(p<0.05)$. Compared with the 1-day $\mathrm{PCl}$, the elongation factor with $\mathrm{SCl}$ increased by $33 \%(p<0.05)$ and $30 \%(p<0.05)$, respectively, and the form factor decreased by $33 \%$ as in the parietal cortex and hippocampus $(p<0.05)$. 
Compared with the control group, the number of hyperchromic neurons in the parietal cortex after 1 hour of SCl significantly increased by $79 \%$, hyperchromic shriveled neurons by $80 \%$, shadow cells by $60 \%$, which is $39 \%, 85 \%$, and $80 \%$ significantly more than in rats with $\mathrm{PCl}$, respectively. In the hippocampus, the number of hyperchromic neurons increased by $77 \%(p<0.05)$, hyperchromic wrinkled cells by $95 \%(p<0.05)$, shadow cells by $100 \%(p<0.05)$, which is more than with PCI by $27 \%(\mathrm{p}<0.05), 85 \%(\mathrm{p}<0.05)$, and $67 \%(p<0.05)$, respectively. There were no differences in the number of pathological forms of neurons between the studied sections of the cortex. By day 1 of $\mathrm{SCl}$, neurons with pericellular oedema $536(536 ; 636.5)$ per $\mathrm{mm}^{2}$ appeared in both studied structures. At the same time, their number in the parietal cortex was significantly higher than in the hippocampus by $25 \%$. In rats with $\mathrm{SCl}$, morphological changes were more pronounced than with $\mathrm{PCl}$, but less than with $\mathrm{SCl}$. The least pronounced changes in size and shape of the neurons were noted in the first subgroup (the interval between CCA dressings was 7 days). The area of the neurons, compared with the control, decreased in the parietal cortex by $39 \%(p<0.05)$, in the hippocampus by $28 \%$ $(p<0.05)$, the elongation factor increased by $8 \%$ $(p<0.05)$, and the form factor decreased by $11 \%$ $(p<0.05)$. When comparing the "PCl" group and the first "SCl" subgroup, the shape of the perikaryon neurons in both studied sections was not significantly different. After 1 day, the sizes of the neurons in this subgroup were $36 \%$ larger $(p<0.05)$ in the parietal cortex and $60 \%(p<0.05)$ in the hippocampus than with $\mathrm{SCl}$, and compared with $\mathrm{PCl}$, smaller by $27 \%(\mathrm{p}<0.05)$ in the parietal cortex. In the hippocampus, the sizes of neuronal perikaryons did not differ from the sizes in the "PCl" group ( $p>0.05)$. In the second and third intervals (between CCA dressings was 3 days and 1 day, respectively), the decrease in the size and deformation of the neuronal perikaryons were significantly more pronounced than in the first subgroup. Compared with the control, the area of the neurons in the second subgroup decreased by $53 \%(p<0.05)$ in the parietal cortex and $40 \%$ in the hippocampus $(p<0.05)$, the elongation factor increased by $14 \%(p<0.05)$, and the form factor decreased by $22 \%(p<0.05)$ in both studied departments $(p<0.05)$.
Compared with the "PCl" in the parietal cortex, the area of neurons decreased by $57 \%(p<0.05)$, and in the hippocampus by $31 \%(p<0.05)$, the form factor by $22 \%(p<0,05)$ and by $17 \%(p<0.05)$, respectively. The elongation factor increased significantly by $14 \%$ and $22 \%$ in the parietal cortex and hippocampus, respectively. Compared with the "SCl" group, no change was observed in the size of the neurons in the parietal cortex, but in the hippocampus, the area in the second subgroup "SSCl" was $15 \%$ larger $(p<0.05)$. The form factor did not change, and the elongation factor significantly increased by $7 \%$ in rats with $\mathrm{SCI}$ both in the parietal cortex and hippocampus. Compared to the characteristics of the neurons in the control, the perikaryon neurons area in the third subgroup SSCl decreased by $60 \%(p<0.05)$ in the parietal cortex and $47 \%$ in the hippocampus $(p<0.05)$. The form factor decreased by $11 \%$ $(p<0.05)$, while the elongation factor increased by $20 \%$ in both studied regions $(p<0.05)$. Compared with the group "PCl", the area of the neurons in the third subgroup with an ischemic period of 1 hour was $65 \%$ less $(p<0.05)$ in the parietal cortex and $40 \%$ in the hippocampus $(p<0.05)$.

The form factor did not change, and the elongation factor increased by $20 \%(p<0.05)$ in both studied departments. In the third subgroup, changes in the size and shape of neurons after 1 hour did not differ from those with $\mathrm{SCl}(\mathrm{p}>0.05)$, but with an ischemic period of 1 day, the area of the neurons was $15 \%$ greater than with $\mathrm{SCl}$, in the parietal cortex $(p<0.05)$ and by $39 \%$ in the hippocampus $(p<0.05)$. As the interval between CCA dressings decreased from 7 days in the first subgroup to 1 day in the third subgroup, more pronounced changes in the size (significant decrease), and shape of the neurons (a significant increase in the elongation factor and decrease in the form factor), and there was also an aggravation of changes in the chromatophilia of their cytoplasm. With SSCl, as the time interval between CCA dressings decreased, the number of normochromic neurons decreased $(p<0.05)$ and the number of hyperchromic shrivelled neurons increased $(p<0.05)$. The maximum number of hyperchromic shrivelled neurons was observed in the third subgroup (with an interval between CCA dressings of 1 day), especially in the parietal cortex $\left(804 / \mathrm{mm}^{2} ; \mathrm{p}<0.05\right)$, which is $42 \%$ more than in the first subgroup $(p<0.05)$. In 
addition, in the $3^{\text {rd }}$ subgroup, one day after ligation of the second CCA, cells with peripheral oedema $(p<0.05)$ appeared in the parietal cortex, which were already present in rats with $\mathrm{SCl}$ after 6 hours of ischemic period. After 1 day, their number in rats with $\mathrm{SCl}$ was higher in the parietal cortex by $53 \%(p<0.05)$. In rats with $\mathrm{TCl}$, the most pronounced changes in size, shape, and degree of chromatophilia of the cytoplasm of neurons were observed.

With $\mathrm{TCl}$ lasting 1 hour, there was a significant decrease in the size of neurons by $74 \%$ and $50 \%$ in the parietal cortex and hippocampus, and with an ischemic period of 1 day, the area of neurons significantly decreased by $83 \%$ and $76 \%$, respectively, which is more than with 1-hour $\mathrm{TCl}$. Compared with 1-hour $\mathrm{TCl}$, the area of neurons in the parietal cortex decreased by $34 \%$ and $42 \%$ in the hippocampus ( $<<0.05$ for both). Aggravation in the shape of neurons was also noted. With an ischemic period of 1 hour, the elongation factor increased by $35 \%(p<0.05)$, while the form factor decreased by $34 \%(p<0.05)$ both in the parietal cortex and hippocampus. Comparing 1-hour ischemia with 24-hour TCl, the elongation factor increased by $25 \%$ in the parietal cortex $(p<0.05)$ and $22 \%$ in the hippocampus $(p<0.05)$. In animals with TCl lasting 1 hour, the majority of the cells in both studied sections of the cortex were hyperchromic wrinkled neurons (their number significantly increased by $96 \%$ compared to the control). Oedematous swollen neurons $(p<0.05)$ appeared in rats after 1 -hour $\mathrm{TCl}$. The number of hyperchromic shrivelled neurons in the $\mathrm{TCl}$ group significantly increased by $80 \%$, and the number of shadow cells significantly decreased by $30 \%$ than in the SIGM group. After 1 day of the ischemic period, cells with pericellular oedema began to predominate in the population of neurons $(p<0.05)$. At the same time, normochromic neurons were absent $(p<0.05)$, and single hyperchromic neurons were observed only with 1 -hour of $\mathrm{TCl}(\mathrm{p}<0.05)$. Compared with the "SCl" group, with 24-hour $\mathrm{TCl}$, the number of hyperchromic shrivelled neurons and shadow cells decreased by $70 \%(p<0.05)$. A large proportion of the cell population consisted of neurons with pericellular oedema (2680 (2479; $3082) / \mathrm{mm}^{2}$ ), while in the $\mathrm{SCl}$ group such cells accounted for only $16 \%$ of the total number of neurons.

\section{Discussion}

In general, the smallest morphological changes in neurons were noted in the "PCl" groups and the first subgroup "SSCl", with an interval between CCA dressings of 7 days. With these $\mathrm{Cl}$ modelling methods, adaptation processes occur that prevent the development of pronounced morphological changes and allow neurons to adapt to moderate hypoxia. For example, in $\mathrm{PCl}$, the absence of pronounced morphological changes in rats is explained by the compensation of blood circulation along the circle of Willis. With SSCl, when the time interval is sufficient for the development of adaptive processes, the productivity of mitochondrial respiration increases $[7,8]$, and possibly activating the production of nitrogen monoxide and hypoxiainduced factor. Modelling of more severe types of ischemic damage leads to pronounced morphological changes in neurons in the parietal cortex and hippocampus of the rat brain - a decrease in their size, deformation of the perikarya, an increase in the degree of neuronal chromatophilia with their simultaneous wrinkling and subsequent death. To the greatest extent, these violations were expressed in the third subgroup of SSCl with the shortest interval between dressings, which was 1 day and $\mathrm{TCl}$. Hyperchromic neurons are regarded as ischemic-altered cells [9]. The appearance of shrivelled dark cells in hypoxic and anoxic conditions is a universal and most severe form of reactive and pathological changes in neurons, accompanied by changes in the metabolic rate, tinctorial properties of the cytoplasm, cell karyoplasm and varying degrees of ultrastructural changes in cytoplasmic organelles. Therefore, in hyperchromic wrinkled neurons, metabolic processes decrease, and the breakdown of nucleoproteins, especially nuclear ones, prevails over their synthesis. The reserves of ribonucleoprotein particles in the nucleus are preserved, but their excretion into the cytoplasm is blocked [10-14]. At later stages of ischemia, swelling of neurons is observed, accompanied by the dissolution of the chromatophilic substance, coarsening, disintegration and melting of neurofibrils, pycnosis of nuclei, and thickening and decay of processes. The neuropil is vacuolated and fragmented, undergoing granular-lumpy disintegration, and myelin dissolves, as a result of which lipid droplets begin to be detected along the nerve fibres [12-14]. 


\section{Conclusion}

When comparing the morphological changes of neurons in the parietal cortex with those in the hippocampus of the rat brain, it can be seen that, with the aggravation of the severity of cerebral ischemia, a progressive increase in the number of hyperchromic shrivelled neurons and neurons with pericellular oedema. The most obvious morphological changes (a decrease in the size and deformation of the perikaryons of neurons) were observed in the conditions of TCl after 1 day. Similar to $\mathrm{TCl}$, but less gross violations, were found with daily $\mathrm{SCl}$ and in the "SSCl" subgroup with an interval between CCA dressings for 1 day. Changes in the parietal cortex and hippocampus were unidirectional, but in the parietal cortex, which is most sensitive to oxygen deficiency, they were more pronounced. The results provide a fundamental basis for further detailing the pathogenesis of ischemic brain damage and searching for an effective correction for this pathology.

\section{Acknowledgements}

\section{Conflict of interest statement}

The authors declare no conflict of interest.

\section{Funding sources}

There are no sources of funding to declare.

\section{References}

1. Sveinsson ÓÁ, Kjartansson Ó, Valdimarsson EM. Heilablóðpurrð / heiladrep. Faraldsfræði, orsakir og einkenni. Læknablaðið. 2014 May 2;2014(05):271279. https://doi.org/10.17992/lbl.2014.05.543

2. Qian $\mathrm{H}$, Zhang H, Yin L, Zhang J. Postischemic Housing Environment on Cerebral Metabolism and Neuron Apoptosis after Focal Cerebral Ischemia in Rats. Current Medical Science. 2018 Aug;38(4):656-665. https://doi.org/10.1007/s11596-018-1927-9

3. Rahaman P, Del Bigio MR. Histology of Brain Trauma and Hypoxia-Ischemia. Academic Forensic
Pathology. 2018 Aug 31;8(3):539-554. https://doi. org/10.1177/1925362118797728

4. Zhang L, Zhang RL, Jiang $Q$, Ding G, Chopp $M$, Zhang ZG. Focal embolic cerebral ischemia in the rat. Nature Protocols. 2015 Mar 5;10(4):539-547. https:// doi.org/10.1038/nprot.2015.036

5. Igorevna Bon L. Effects of Experemental Cerebral Ishemia on Metabolic Characteristics of Parietal Cortex Neurons. Bioprocess Engineering. 2018;2(1):1. https://doi.org/10.11648/j.be.20180201.11

6. Paxinos G, Watson C. The Rat Brain in Stereotaxic Coordinates. Australia: Academic Press; 1998.

7. Bon L, Maksimovich N, Zimatkin S. Changes in the immunoreactivity of ATP synthase and chromatophilia of the cytoplasm of neurons in the parietal cortex of rats under conditions of stepwise cerebral ischemia. Orenburg Medical Bulletin. 2020;4:26-31.

8. Maksimovich N, Bon L, Dremza I. Structural and functional features of mitochondria and methods of their study in experiment. Medicus. 2019;5:8-18.

9. Zimatkin SM, Bon' El. Dark Neurons of the Brain. Neuroscience and Behavioral Physiology. 2018 Oct;48(8):908-912. https://doi.org/10.1007/s11055018-0648-7

10. Kherani ZS, Auer RN. Pharmacologic analysis of the mechanism of dark neuron production in cerebral cortex. Acta Neuropathologica. 2008 Jun 3;116(4):447-452. https://doi.org/10.1007/s00401008-0386-y

11. Capo I, Lalosević D. [Interpretation of dark neurons in experimental model of ischemia, neurointoxication and brain infection]. Med Pregl. 2011 Jan-Feb;64(12):101-6. PMID 21548279

12. Gallyas F, Pál J, Bukovics P. Supravital microwave experiments support that the formation of "dark" neurons is propelled by phase transition in an intracellular gel system. Brain Research. 2009 May;1270:152156. https://doi.org/10.1016/j.brainres.2009.03.020

13. Morphological disturbances of rat parietal cortex and hippocampus neurons in the dynamics steady subtotal ischemia of the brain. Сибирский научный медицинский журнал. 2020;(2). https:// doi.org/10.15372/ssmj20200204

14. Bon LI, Maksimovich NYe, Zimatkin SM. Morphological disorders of neurons in the hippocampus of rats with subtotal and total ischemia. Orenburg Medical Bulletin. 2020;2:41-6. 